\title{
Imaging in lung transplants: Checklist for the radiologist
}

\author{
Rachna Madan, Thanissara Chansakul'1, Hilary J Goldberg² \\ Departments of Thoracic Imaging, 'Radiology and 'Medicine, Lung Transplant Program, Brigham and Women's Hospital, Harvard \\ Medical School, Massachusetts, USA
}

Correspondence: Dr. Rachna Madan, 20 Stearns Road, Apartment 41, Brookline, Massachusetts - 02446, USA.

E-mail: rmadan@partners.org

\begin{abstract}
Post lung transplant complications can have overlapping clinical and imaging features, and hence, the time point at which they occur is a key distinguisher. Complications of lung transplantation may occur along a continuum in the immediate or longer postoperative period, including surgical and mechanical problems due to size mismatch and vascular as well as airway anastomotic complication, injuries from ischemia and reperfusion, acute and chronic rejection, pulmonary infections, and post-transplantation lymphoproliferative disorder. Life expectancy after lung transplantation has been limited primarily by chronic rejection and infection. Multiple detector computed tomography (MDCT) is critical for evaluation and early diagnosis of complications to enable selection of effective therapy and decrease morbidity and mortality among lung transplant recipients.
\end{abstract}

Key words: Acute and chronic rejection; anastomotic complications; lung transplant

\section{Introduction}

Lung transplantation is an accepted therapeutic option for patients with end-stage lung disease. The indications for transplantation span the spectrum of pulmonary diseases [Table 1]. Three transplantation procedures are commonly performed: Single-lung transplantation (SLT), bilateral lung transplantation (BLT), and heart-lung transplantation. BLT is required for patients with bronchiectasis including cystic fibrosis and for patients with significant pulmonary arterial hypertension. Either BLT or SLT may be performed in patients with diseases other than bronchiectasis and pulmonary hypertension, unless special considerations dictate the use of BLT. Nevertheless, over the past decade, BLT has been increasingly used due to better long term survival, particularly among patients with chronic obstructive lung disease (COPD). ${ }^{[1-3]}$

\begin{tabular}{|l|l|}
\hline \multicolumn{2}{|c|}{ Access this article online } \\
\hline Quick Response Code: & \\
\cline { 1 - 2 } & Website: \\
\hline & www.ijri.org \\
& DOI: \\
\hline
\end{tabular}

Heart-lung transplantation was traditionally required for secondary pulmonary hypertension due to cardiac causes (Eisenmenger syndrome). However, with improvements in supportive technology and experience with transplant procedure, heart-lung transplantation has become very infrequent.

Survival rates for lung transplant patients remain fairly low, with the median survival after lung transplantation being 5.3 years. ${ }^{[4]}$ Although lung transplantation is a well-established procedure, complications are frequent. Life expectancy after lung transplantation has been limited primarily by chronic rejection and infection. ${ }^{[4]}$ Post-transplantation management comprising monitoring allograft function, regulating immunosuppressive regimen, as well as detecting and treating complications expeditiously is crucial to optimize patient outcome.

Early and accurate diagnosis of complications can be challenging. Causes of complications are varied, ranging from technical problems to host immune response and predisposition to infections. Several of these complications may coexist, resulting in complex radiologic pictures. Given the degree of complexity, an interdisciplinary approach is important. Bronchoscopy, bronchoalveolar lavage, and transbronchial biopsy may be required to identify 
complications. Radiologist's awareness of complications and associated imaging features that may occur at varying time points following transplantation is essential to effectively guide clinical management.

This article will illustrate imaging features of a spectrum of complications that may occur following lung transplantation. As many of these complications can have overlapping clinical and imaging features, the time point at which they occur is a key distinguisher. Post lung transplantation complications are categorized according to the temporal relationship related to the operation as immediate $(<24 \mathrm{~h})$, early ( $>24$ h-1 week), intermediate (8 days-2 months), primary late (2-4 months), and secondary late ( $>4$ months) complications. ${ }^{[5]}$ Table 2 lists a comprehensive, but by no means complete checklist of complications which can assist the radiologist and clinician in evaluating a patient following

Table 1: Indications for lung transplantation (in order of frequency) Chronic obstructive pulmonary disease

Idiopathic pulmonary fibrosis

Alpha 1-antitrypsin deficiency emphysema

Cystic fibrosis

Idiopathic pulmonary arterial hypertension

Others

Sarcoidosis, interstitial lung disease, secondary pulmonary hypertension, lymphangioleiomyomatosis, Langerhan's cell histocytosis, re-transplantation

Table 2: Checklist of complications following lung transplantation based on time of occurrence

\begin{tabular}{ll}
\hline $\begin{array}{l}\text { Time period } \\
\text { post-transplantation }\end{array}$ & Complications \\
\hline Immediate (<24 h) & $\begin{array}{l}\text { Donor-recipient mismatch } \\
\text { Hyperacute rejection }\end{array}$ \\
$\begin{array}{ll}\text { Early } & \text { Primary graft dysfunction (reperfusion edema or ischemia- } \\
& \text { reperfusion injury) } \\
& \text { Pleural complications } \\
& \text { Pleural effusion } \\
& \text { Pneumothorax } \\
& \text { Hemothorax } \\
& \text { Empyema } \\
\text { Intermediate } & \text { Air leak } \\
\text { (8 days-2 months) to } & \text { Acute rejection } \\
\text { primary late } & \text { Anastomotic complications } \\
\text { (2-4 months) } & \text { Airway and bronchial anastomotic complications } \\
& \text { (stenosis, dehiscence, infection) } \\
& \text { Vascular anastomotic complications (stenosis or occlusion) } \\
& \text { Infections } \\
& \text { Pulmonary thromboembolic events } \\
& \text { Chronic rejection } \\
\text { Secondary late } & \text { Upper lobe fibrosis and pulmonary pleuroparenchymal } \\
\text { (>4 months) } & \text { fibroelastosis } \\
& \text { Cryptogenic organizing pneumonitis } \\
& \text { Post-transplant lymphoproliferative disorder } \\
\text { Recurrence of primary disease } \\
\text { Transbronchial biopsy-associated complications }\end{array}$ \\
&
\end{tabular}

lung transplantation. Use of problem-specific multiple detector computed tomography (MDCT) airway protocols is also discussed for evaluation of unique complications such as bronchiolitis obliterans and bronchomalacia.

\section{Immediate complications ( $<24 \mathrm{~h}$ )}

\section{Donor-recipient size mismatch}

The donor and recipient are matched for size preoperatively. Size matching is gauged primarily by body height; however, thoracic volume is somewhat pliable and some size discrepancies do not affect overall survival or pulmonary function, and therefore are acceptable. ${ }^{[6]}$ If the donor lung is too large for the recipient, passive atelectasis may manifest on an immediate postoperative chest radiograph. In addition to decreased pulmonary function in the immediate post-transplantation period, superimposing complications in the atelectatic lung may result in scarring and further reduction in lung volume. Donor lungs that are considered too large may require surgical downsizing prior to transplantation. At the other extreme, an undersized donor lung can result in mechanical problems related to residual space, such as intractable pleural effusion or pneumothorax. When a small donor lung is used in single transplantation for emphysema, hyperexpansion of the native emphysematous lung may cause compression of the transplanted lung, thereby inhibiting its function. In this situation, surgery to reduce the native lung volume or bullectomy may prove beneficial. ${ }^{[7]}$

\section{Hyperacute rejection}

Hyperacute rejection is an immediate, complement-mediated injury that leads to acute onset of alveolar damage, resulting in graft dysfunction or failure within minutes to hours following graft reperfusion. It occurs when the preformed an ti-human leukocyte antigen (anti-HLA) or anti-ABO antibody in the recipient reacts with a corresponding or cross-reactive antigen in the donor organ. With detailed attention to $\mathrm{ABO}$ compatibility and human leukocyte antigen (HLA) characteristics of the donor and recipient, this phenomenon has become exceedingly rare. On radiograph, hyperacute rejection manifests as dense homogeneous opacification throughout the allograft. ${ }^{[8-10]}$ Management options are limited, but may include aggressive immunosuppression, plasmapheresis, and urgent retransplantation. ${ }^{[8]}$

\section{Early complications ( $>24$ h-1 week)}

Primary graft dysfunction (reperfusion edema or ischemia-reperfusion injury)

Primary graft dysfunction (PGD) is a nonspecific, non-cardiogenic acute lung injury characterized by diffuse alveolar damage and increased vascular permeability. PGD results from insults that are inherent in the transplantation process, including donor lung ischemia, organ procurement and preservation techniques, and organ implantation and reperfusion. ${ }^{[11]}$ PGD appears within $72 \mathrm{~h}$ of transplantation, peaks in severity on postoperative day 4 , and generally 
improves by the end of the first week. ${ }^{[12]}$ Radiographic and CT manifestations are variable and nonspecific, but most commonly comprise middle and lower lobe predominant airspace opacities. ${ }^{[12]}$ Mixed airspace and interstitial opacities with bronchial and vascular wall thickening may be seen. ${ }^{[5,12]}$ Hyperacute and acute rejection should be considered in the diagnostic evaluation of PGD. Management typically remains largely supportive and follows the paradigm used in acute respiratory distress syndrome (ARDS). ${ }^{[12]}$

\section{Pleural complications}

Acute pleural complications including pleural effusion, pneumothorax, hemothorax, empyema, and air leaks occur in $22-34 \%$ of patients following lung transplantation [Figure 1]. ${ }^{[13,14]}$ Development of early pleural effusion is thought to be due to ischemia, denervation, and subsequent reperfusion of the allograft or by disruption of the pulmonary lymphatics. ${ }^{[13]}$ Pleural effusion recalcitrant to chest tube drainage should raise suspicion for other causes such as empyema, chylothorax secondary to severed thoracic duct, and heart failure. ${ }^{[15]}$ Persistent pneumothorax beyond the early postoperative period raises concern for underlying bronchial anastomotic complications. CT is often helpful in characterizing and guiding management of complex pleural processes.

Intermediate complications ( 8 days- 2 months) and primary late complications (2-4 months)

\section{Acute rejection}

Acute rejection is due to cell-mediated immune response. Approximately half of patients have at least one episode

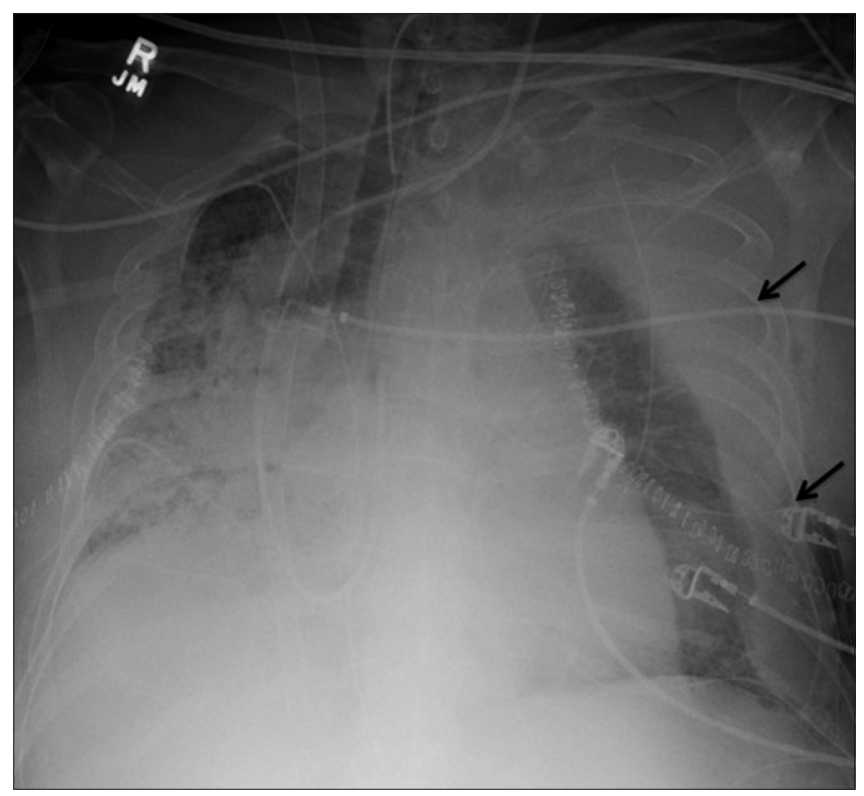

Figure 1: Hemothorax. Chest radiograph day 1 following left lung transplantation and 3 weeks following right lung transplantation. A moderate-to-large left pleural fluid collection surrounding the allograft, representing hemothorax (arrows), necessitating emergent thoracotomy for evacuation of hematoma. Dense consolidative opacities in the right transplant represent pneumonia and pulmonary infarcts of acute rejection in the first year following transplant. ${ }^{[16]}$ Recurrent acute rejection is a risk factor for the development of chronic rejection. ${ }^{[16]}$

Acute rejection has a radiographic appearance similar to PGD; airspace abnormalities such as groundglass opacities with accompanying septal thickening and pleural effusion may be seen Figure 2. ${ }^{[17]}$ Of note, patients may present with subclinical acute rejection in the absence of imaging abnormalities. Significant improvement of radiographic abnormality following intravenous steroid administration within 48-72 $\mathrm{h}$ favors diagnosis of acute rejection..$^{[18]}$

\section{Anastomotic complications}

Lung transplantation involves three different anastomoses: Airway, pulmonary arterial, and pulmonary vein to left atrium.

\section{Airway and bronchial anastomotic complications}

Commonly seen airway complications include stenosis and airway infection. The prevalence is approximately $15 \% .{ }^{[19]}$ Dehiscence is a rare airway complication of transplantation. Dehiscence and infection are seen earlier in the postoperative period ( 1 week- 2 months), while stenosis and bronchomalacia are seen later at 2-4 months post procedure. Key risk factors predisposing to airway-related complications include donor bronchus ischemia caused by disruption of native bronchial circulation, followed by recurrent rejection and infection. There has been a decrease in airway complications following refinements in surgical techniques and immunosuppressive therapy. Advances in MDCT technology and newer flexible ultrathin bronchoscopic techniques have facilitated early detection and management of airway anastomotic

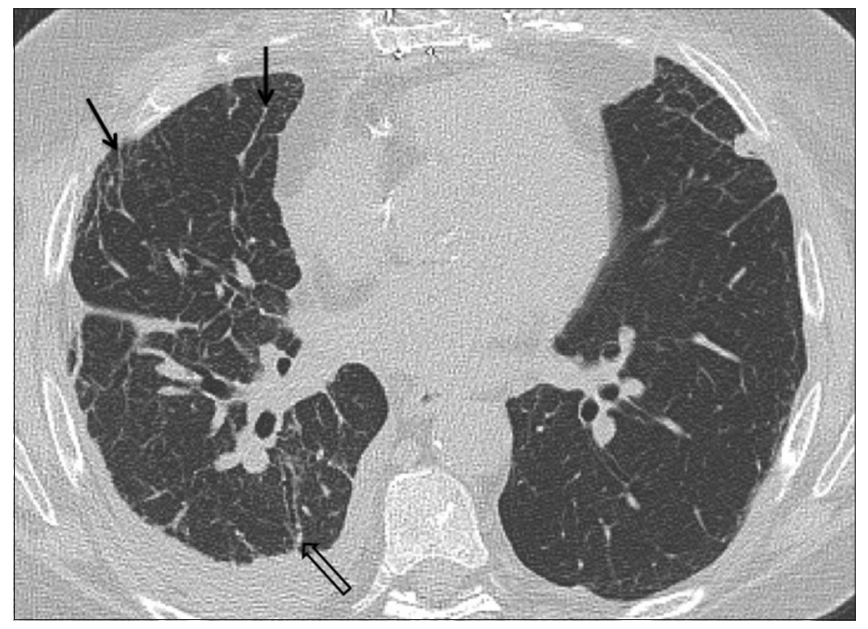

Figure 2: Acute rejection. Axial CT in lung windows following bilateral lung transplantation reveals right-sided septal thickening (arrows) and subtle groundglass, peribronchial cuffing and bronchiectasis (open arrow) and a small right effusion. Transbronchial biopsy was consistent with rejection. Transplanted left lung was normal 
complications. ${ }^{[20]} \mathrm{CT}$ can demonstrate focal mucosal irregularities, necrosis, and debris formation at an advanced stage, whereas findings are seen much earlier on bronchoscopy.

Partial dehiscence is more common than complete dehiscence, and presents as enlarging ipsilateral pneumothorax or pneumomediastinum. MDCT with thin section curved planar reformations may show focal bronchial wall defects and perianastomotic air collections [Figure 3]. Most of these are managed conservatively as they may resolve spontaneously. It is important to be aware of the anastomotic surgical technique used as the telescoping bronchus may mimic dehiscence [Figure 4]. This involves end-to-end anastomosis of the posterior membranous portion of the larger bronchus and invagination of the cartilaginous portion of the smaller bronchus into the larger bronchus. The telescoped segment has the appearance of a bronchial wall defect or small tubular extraluminal gas collection. Curved planar reformations are exquisite in separating telescoping bronchus from dehiscence. ${ }^{[21]}$

Anastomotic stenosis is more common than dehiscence, and can be seen later in the postoperative period. Thin section axial and curved planar reformations as well as virtual bronchoscopy may show areas of fixed focal narrowing and irregularity [Figure 4]. Alternatively, patients may present with recurrent lobar collapse [Figure 5], in which case proximal airway stenosis should be actively assessed for. Management includes debridement of granulation tissue, balloon dilation, and/ or stent placement.

Transient airway narrowing or dynamic bronchomalacia can be detected on bronchoscopy as well as on CT. Paired end-inspiratory and dynamic expiratory CT images are compared to identify if there is greater than $50 \%$ reduction in airway diameter or lunate shape of the airway. ${ }^{[22]}$

\section{Vascular anastomotic complications}

Post lung transplant arterial obstruction is more common than venous obstruction. Common causes of narrowing include excessive length of vascular pedicle, short allograft artery length, and presence of restrictive suture and clot. Pulmonary artery stenosis can occur early and late after lung transplantation. ${ }^{[23]}$ Patients may present with shortness of breath, hypoxia, signs of pulmonary hypertension, and right heart failure, and non-resolving pulmonary opacities secondary to infarction on radiographs and CT [Figure 6]. Contrast-enhanced CT angiogram may show narrowing or occlusion leading to non-resolving pulmonary opacities secondary to lung infarction or anastomotic dehiscence leading to catastrophic hemothorax. Treatment includes angioplasty and stenting.

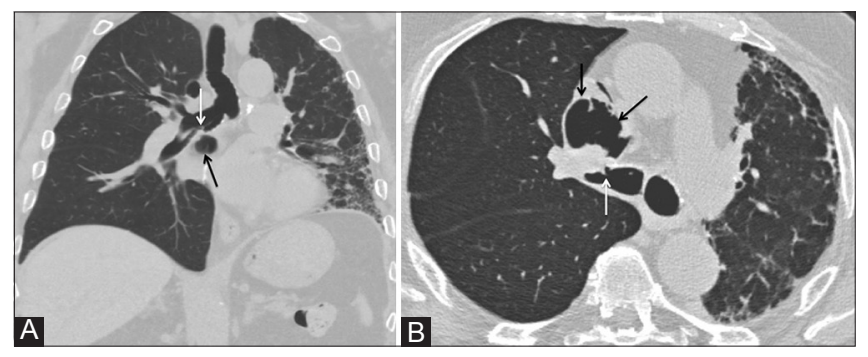

Figure 3 ( $A$ and $B$ ): Partial dehiscence following right lung transplantation for usual interstitial pneumonitis (UIP). (A and B) Coronal and axial CT images in lung windows show focal air collection in mediastinum adjacent to anastomosis (black arrows). No direct communication is identified; however, the location and bronchial mucosal irregularity (white arrows) suggest underlying partial dehiscence

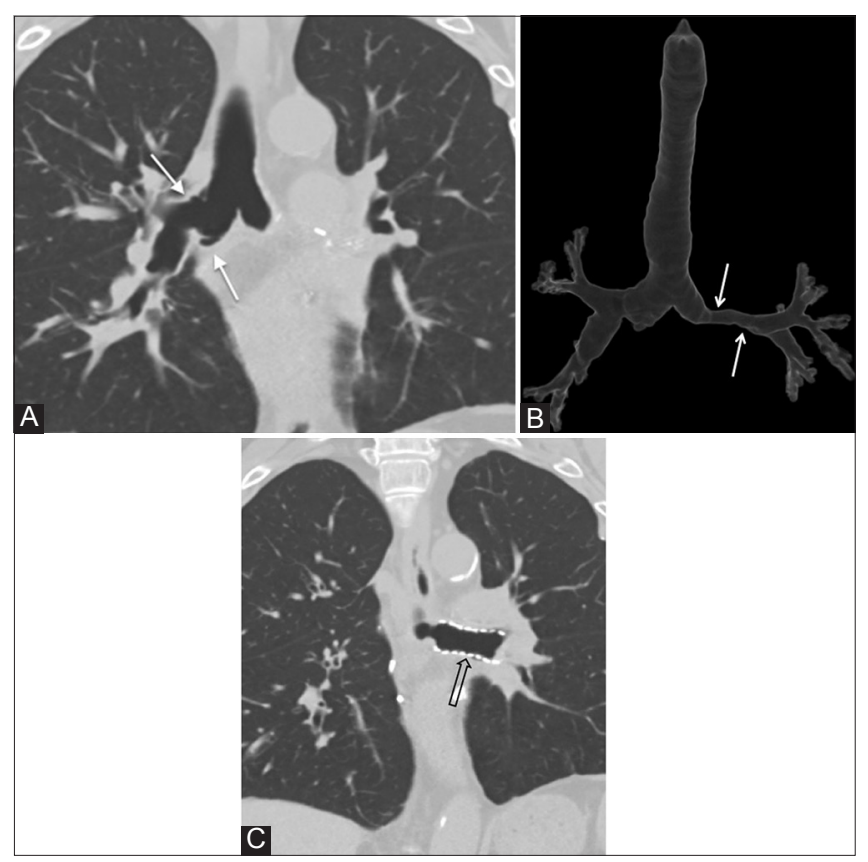

Figure 4 (A-C): Post bilateral lung transplant for cystic fibrosis. (A) Coronal CT in lung windows shows telescoping anastomosis on the right, a normal postoperative appearance (white arrows) (B) Coronal 3D volume-rendered image reveals smooth long segment narrowing of left mainstem bronchus (white arrows), requiring subsequent airway stent placement (open arrow) (C)

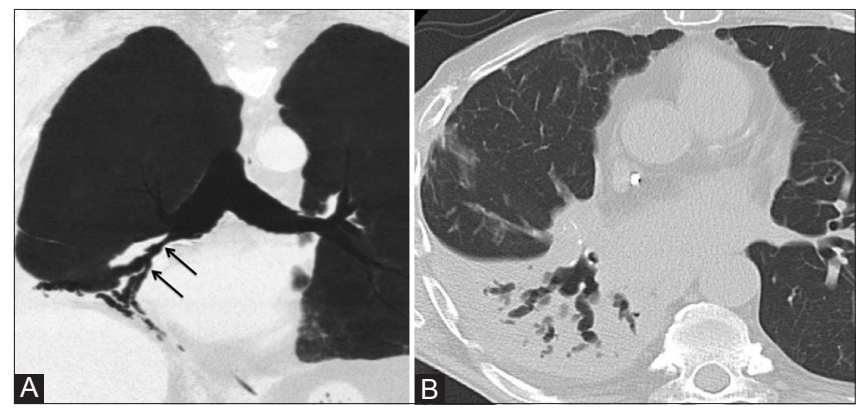

Figure 5 (A and B): Right lung transplant with recurrent right lower lobe collapse. (A) Coronal minimum intensity projection (minIP) CT image demonstrates marked fixed narrowing and irregularity of bronchus intermedius and right lower lobe (RLL) bronchus (arrows) (B) Distal RLL collapse and varicose bronchiectasis due to postobstructive changes 


\section{Pulmonary infections}

Pulmonary infections are an important cause of morbidity and mortality. Certain infections are more commonly seen than others in a given postoperative period [Table 3]. CT is useful in narrowing the differential diagnosis, identifying the severity of infection, and selecting the best site for bronchoscopy or percutaneous sampling.

Bacterial infections, particularly due to Gram-negative bacteria such as Pseudomonas and Klebsiella species, as well as Staphylococcus aureus, are fairly common within the first month after transplantation. ${ }^{[5]}$ CT may show patchy and confluent consolidation with air bronchograms and multifocal tree-in-bud nodularity [Figure 7]..$^{5]}$

Fungal infection is a common pulmonary complication of lung transplant. Aspergillus fumigatus is the most common cause of fungal pneumonia and can also cause airway infections. It is frequently difficult to differentiate colonization from true invasive disease. Infection may lead to ulcerative tracheobronchitis, anastomotic infection, or angioinvasive fungal infection with solid nodules demonstrating the halo sign [Figure 8]. Candidal infections can be seen early in the postoperative course and can cause a range of abnormalities including pneumonia, mediastinitis, and esophagitis. Besides multifocal mass-like consolidation, nodules may also be seen.

Cytomegalovirus (CMV) pneumonitis is the most common manifestation of CMV infection and usually occurs between 1 and 6 months after transplant. ${ }^{[2]}$ The

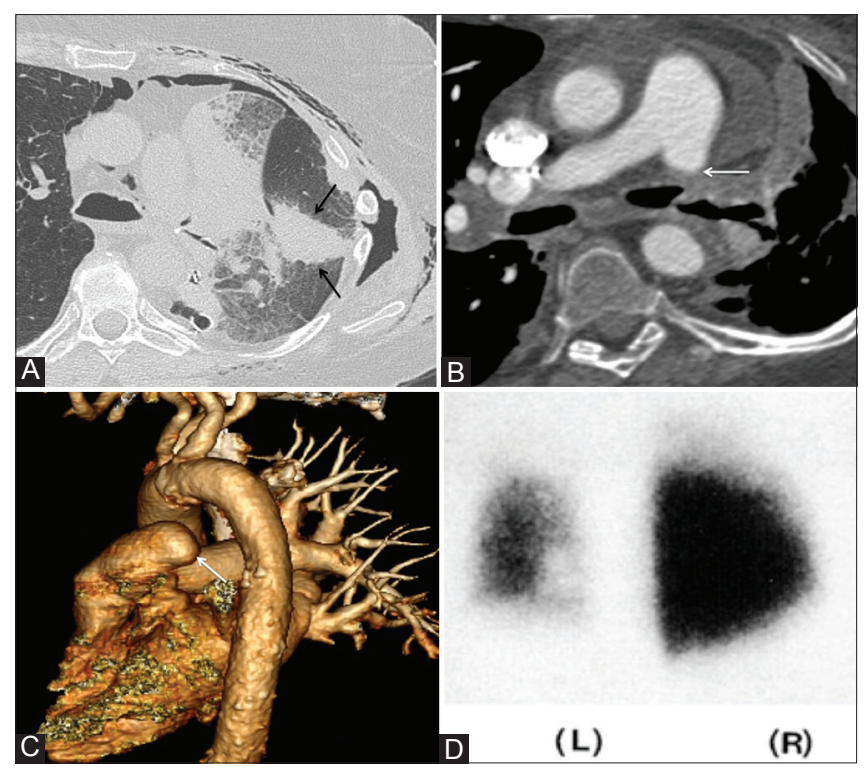

Figure 6 (A-D): Vascular anastomotic complication. (A) Post left lung transplant with peripheral wedge-shaped multifocal consolidation in transplanted left lung is suspicious for an infarct (black arrows). (B and C) Axial CECT and 3D colored volume-rendered image shows abrupt occlusion of proximal left main pulmonary artery (LPA) (white arrows) and no LPA branch flow (D) Perfusion scan shows only $3 \%$ flow to the right lung incidence has decreased due to effective prophylaxis. The risk of infection is highest in seronegative recipients who receive seropositive donor lungs. Imaging features include geographic groundglass opacities with crazy paving appearance, septal thickening and bronchial wall thickening, and centrilobular nodules. Besides being associated with immediate morbidity and mortality, these infections are associated with development of bronchiolitis obliterans. ${ }^{[25]}$

\section{Pulmonary thromboembolic events}

The first few months following transplant surgery are associated with increased thrombogenesis related to increased perfusion of the allograft and arterial anastomosis. Since most CT imaging in the post-transplant period is done without contrast to protect the kidneys in these patients receiving immunosuppressive therapy, a high level of suspicion is needed to request a CT pulmonary angiogram [Figure 9A]. Development of embolism may be a catastrophic event in this population, leading to rejection and rapid decline in lung function [Figure 9B].

\section{Secondary late complications ( $>4$ months)}

\section{Chronic rejection}

Chronic lung allograft dysfunction (CLAD) remains the major late complication of lung transplantation, affecting at least $50 \%$ of recipients at 5 years. This complication can be seen in the first post-transplant year in $7-10 \%$ of patients. Bronchiolitis obliterans, which involves scarring of the

\section{Table 3: Types of infections and typical time of occurrence}

\begin{tabular}{lc}
\hline Pathogen & Typical time of occurrence \\
\hline $\begin{array}{l}\text { Bacterial infections } \\
\text { Fungal infections }\end{array}$ & 1 month \\
$\quad$ Candida & \\
$\quad$ Aspergillus & $1-3$ months \\
Viral & $1-6$ months \\
$\quad$ Cytomegalovirus & \\
$\quad$ Respiratory syncytial virus, parainfluenza, & $1-6$ months \\
$\quad$ adenovirus & $2-24$ months \\
\hline
\end{tabular}

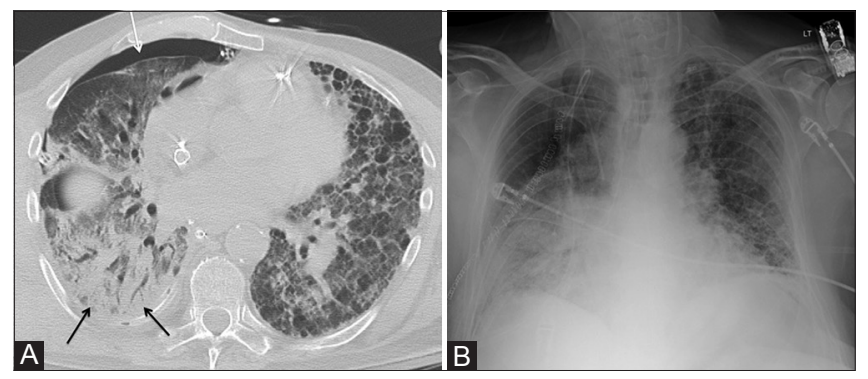

Figure 7 (A and B): Transplant complicated by pneumonia. (A) Radiograph demonstrates consolidative opacity in right middle and lower lobes. Sputum culture grew Serratia marcescens (B) Noncontrast chest CT in lung windows demonstrated dense consolidative opacity in right lower lobe, representing pneumonia (black arrows). Note small right pneumothorax (white arrow). Fibrosis is seen in the native left lung. Emergent left lung transplantation was needed due to acute respiratory failure 
smaller distal airways, is the hallmark of classically defined chronic rejection. ${ }^{[26]}$ Risk factors include multiple episodes of acute rejection, infection, and gastroesophageal reflux. The diagnosis is established by pulmonary function testing and graded according to International Society for Heart and Lung Transplantation criteria, or by lung biopsy. ${ }^{[27,28]}$ Frequently, patients with CLAD may have a combined restrictive obstructive defect on pulmonary function testing and corresponding CT correlate such as apical pleuroparenchymal fibroelastosis (PPFE) and organizing pneumonitis.

Chest radiographs may show chronic airspace opacities with architectural distortion, patchy hyperinflation, subsegmental atelectasis, and bronchiectasis. ${ }^{[29]}$ HRCT findings include bronchiectasis and bronchial wall thickening, mosaicism (mixed hypo- and hyperattenuation) and air trapping, nodular opacities, as well as distortion of bronchovascular structures. If there is a clinical suspicion for chronic rejection, paired inspiratory and end-expiratory $\mathrm{CT}$ images can be acquired and compared to evaluate for air trapping due to bronchiolitis obliterans, though the sensitivity and specificity of this modality is limited. Presence of air trapping is a specific $\mathrm{CT}$ sign and strong correlate of bronchiolitis obliterans. ${ }^{[30]}$

\section{Upper lobe fibrosis and PPFE}

Progressive upper lobe fibrosis can be seen 1-4 years after transplantation. Imaging findings include coarse septal thickening, reticular abnormality, traction bronchiectasis, honeycombing, architectural distortion, and loss of volume. Recently, pleuroparenchymal fibroelastosis (PPFE) has been described in patients with lung transplant and bone marrow transplant as a cause of restrictive allograft syndrome. This is characterized by apical pleural fibrosis and subjacent parenchymal fibroelastosis with a sharp demarcation between areas of fibroelastosis and uninvolved parenchyma. CT reveals severe pleural and subpleural thickening with fibrotic changes in the subjacent parenchyma with associated traction bronchiectasis and honeycombing [Figure 10].

\section{Cryptogenic organizing pneumonitis}

Organizing pneumonitis can be associated with both acute and chronic rejection. It can be seen in patients with acute rejection, presenting as peribronchial, subpleural, or perilobular areas of consolidation, with architectural distortion [Figure 11]. ${ }^{[31]}$ Frequently, a reverse halo sign and arc-shaped opacities may be seen. Bronchoscopy is an important step in the management of these patients to exclude infection before starting high-dose steroids, following which there is often dramatic improvement in the pulmonary opacities.

\section{Post-transplant lymphoproliferative disorder}

Post-transplant lymphoproliferative disorder (PTLD) is uncommon and occurs in $<6 \%$ of patients, with most common risk factors being immunosuppression with cyclosporine and Ebstein-Barr virus infection. ${ }^{[32]}$ Intrathoracic disease is most common in the first year after transplant, while extrathoracic disease is seen later in the post-transplant

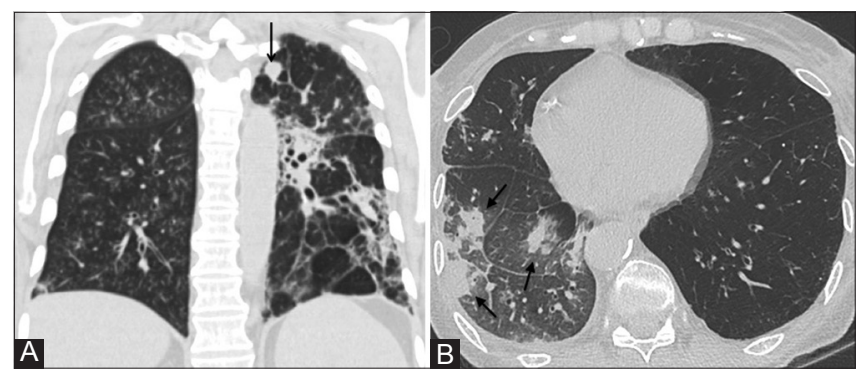

Figure 8 (A and B): Invasive aspergillosis infection secondary to transplant-associated immunosuppression. CT scans in lung windows. (A) New left apical nodule in native left lung (arrow) was biopsied and consistent with Aspergillus infection. Surrogate fungal markers galactomannan and $\beta$-D-glucan were positive. Diffuse centrilobular nodules in right lung due to infectious bronchiolitis. (B) Multifocal nodular, mostly peripheral nodular, consolidation (arrows) and perilesional groundglass demonstrated no improvement following treatment with antibiotics. Diagnosis consistent with angioinvasive Aspergillus pneumonia

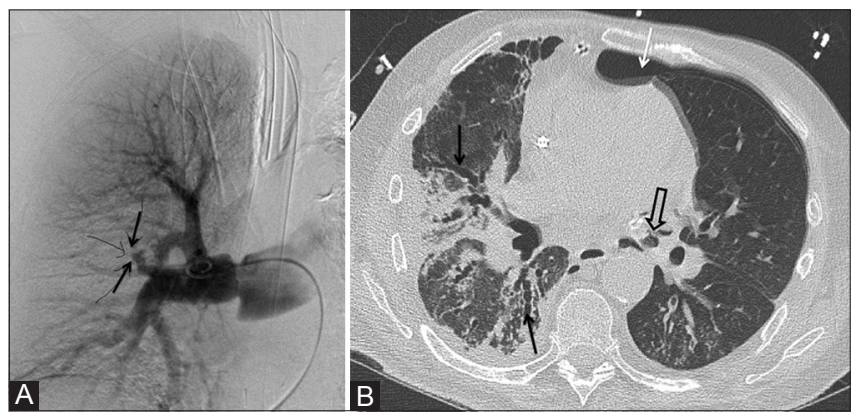

Figure 9 (A and B): Acute-on-chronic rejection due to pulmonary embolism and pneumonia in transplanted right lung. (A) Pulmonary angiogram shows filling defect in right middle lobe artery (arrows) due to embolus. (B) CT in lung windows few weeks later demonstrates peribronchial and subpleural consolidation and traction bronchiectasis in right lower lobe (black arrows), showing rejection and infection. Irregularities of left bronchial anastomosis with adjacent small pneumomediastinum (black open arrow) and small left pneumothorax (white arrow) due to left bronchial anastomotic dehiscence
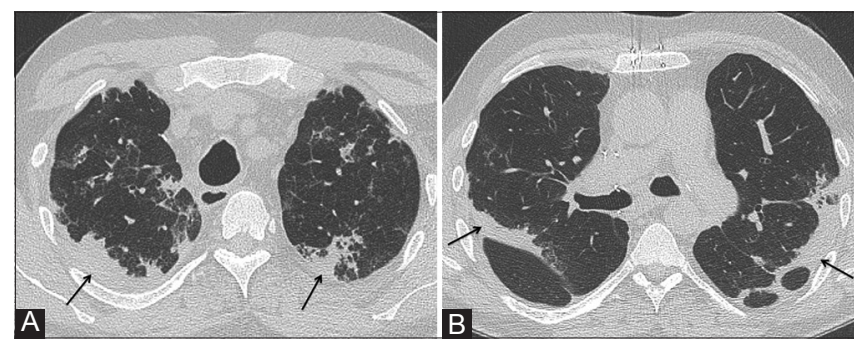

Figure 10 (A and B): Chronic lung allograft dysfunction (CLAD) with both obstructive and restrictive features in a setting of declining pulmonary function. ( $A$ and $B$ ) Chest $C T$ images in lung windows demonstrate apical peripheral subpleural areas of consolidation with associated groundglass opacities and architectural distortion (arrows). Wedge biopsy showed organizing pneumonia and pleuroparenchymal fibroelastosis (PPFE) 
period. Early disease responds more frequently to antiviral therapy and reduction of immunosuppression, while late disease may require chemotherapy and irradiation.

MDCT and 18-fluorodeoxyglucose positron emission tomography/computed tomography (18F-FDG PET/ CT) are well-suited for evaluation of these patients. Well-circumscribed pulmonary nodules or mass-like consolidation can be seen on CT. Frequently, multistation mediastinal, hilar, and extrathoracic adenopathy is identified on cross-sectional imaging [Figure 12]. Biopsy may be needed to differentiate infectious nodules from those secondary to PTLD, as well as to rule out other causes of malignancy besides PTLD. Extrathoracic disease may involve the gastrointestinal tract, skin, or oropharynx. ${ }^{[32]}$

\section{Recurrence of primary disease}

Sarcoidosis is the most commonly recurrent primary disease, at approximately $35 \% .{ }^{[33]}$ Lymphangioleiomyomatosis may also recur in the allograft. The imaging features are specific to the recurrent disease, and these findings may be seen anytime during the course of disease.

\section{Transbronchial biopsy-associated complications}

Bronchoscopy with transbronchial biopsy aids in the diagnosis of rejection and infection with low overall complication rates between $6 \%$ and $12 \% \cdot{ }^{[34]}$ These include hemorrhage, pulmonary laceration, air-filled cysts, pneumothoraces, and infection. Focal dense parenchymal opacities or consolidation may be seen at sites of biopsy, usually located within $2 \mathrm{~cm}$ of the pleura. ${ }^{[34]}$

\section{Tailoring of MDCT protocols to clinical question}

Most surveillance transplant chest imaging is done using chest radiographs and non-contrast CT. Problem-specific protocols should be utilized to answer specific clinical questions [Figure 12]. High-resolution computed tomography (HRCT) images allow early and more sensitive assessment of interstitial thickening, reticulation, and bronchiectasis, all of which are imaging markers of rejection. In suspected chronic allograft dysfunction, end-expiratory images should be obtained in addition to inspiratory images to assess for air trapping, an imaging marker for bronchiolitis obliterans. In patients with suspected dynamic airway narrowing or bronchomalacia, dynamic expiratory CT images should be obtained to look for abnormal compliance and collapsibility. ${ }^{[35]}$ The $3 \mathrm{D}$ reformations and curved planar reformats create a roadmap for the bronchoscopist and the surgeon for sampling of focal lesions and stent placement. Contrast-enhanced CT angiogram is needed in patients with suspected pulmonary embolism and vascular anastomotic complications.

In patients with new nodules or mass and increasing adenopathy, PTLD as well as primary lung malignancy or atypical infection is a concern. FDG PET/CT is well-suited for defining the extent of disease and selecting an appropriate site for tissue sampling [Figure 13].

Imaging also plays a key role in appropriate patient selection prior to lung transplant. While a chest CT is routinely done prior to transplant to rule out an active infection or developing malignancy in these patients, other tests are chosen based on the underlying disease such as

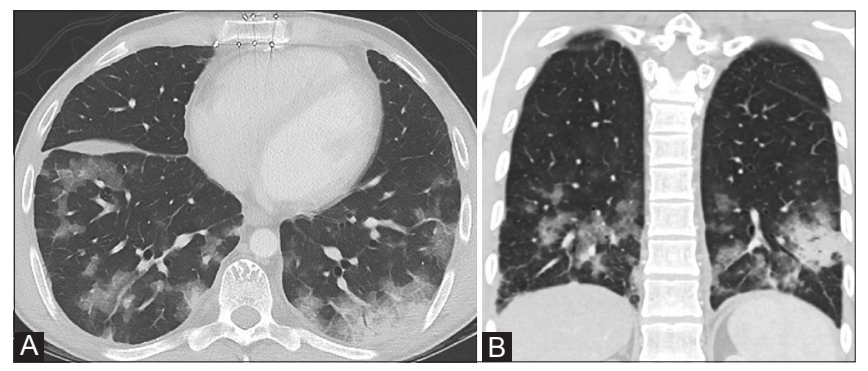

Figure 11 (A and B): Bilateral lung transplant complicated by rejection and organizing pneumonitis. Axial (A) and coronal (B) CT images in lung windows demonstrate basilar subpleural and peribronchial consolidation and groundglass opacities with early architectural distortion. Imaging features consistent with organizing pneumonia. Bronchoscopy was negative for infection. Abnormality responded to treatment with pulsed steroids

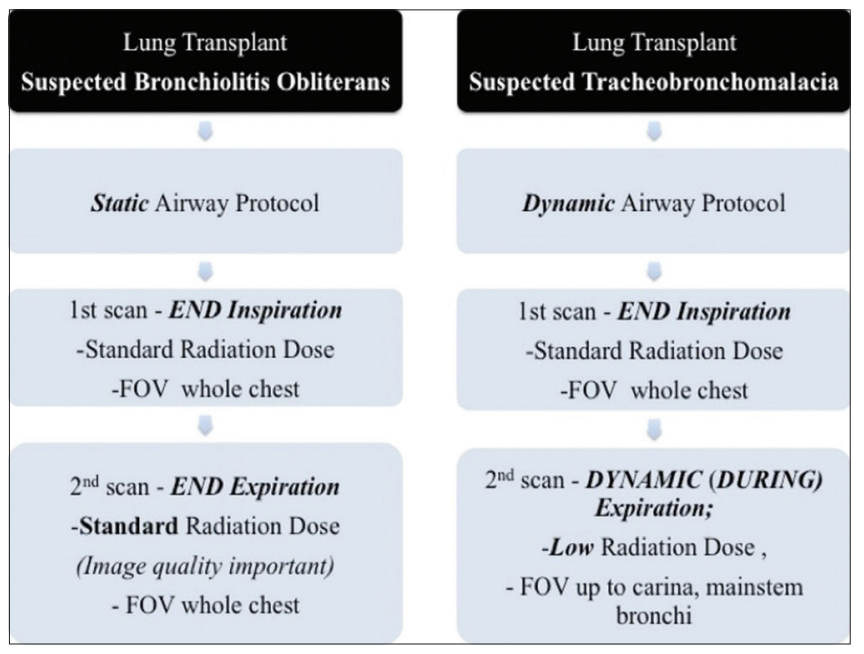

Figure 12: Problem-specific MDCT protocols for evaluation of patients following lung transplantation
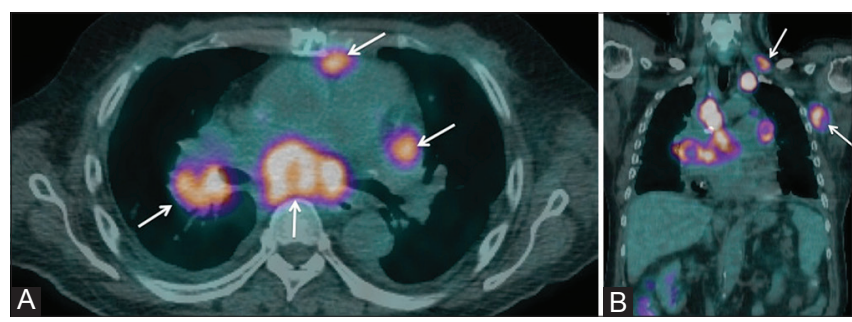

Figure 13 (A and B): Post-transplant lymphoproliferative disorder (PTLD) 2 years following lung transplant. Axial (A) and coronal (B) Fused 18F-FDG PET/CT images demonstrate multistation nodal enlargement associated with intense tracer uptake within the mediastinum, bilateral hila, left supraclavicular region, and left axilla (arrows). Biopsy was consistent with PTLD 
Madan, et al:: Imaging in lung transplants: Checklist for the radiologist

\begin{tabular}{|l|l|}
\hline Diagnostic Test & Timing of Test \\
\hline ECHO & Within 1 year \\
\hline Right Heart Catheterization & Within 5 years \\
\hline Left Heart Catheterization & If age above 40 within 5 years \\
\hline EKG & \\
\hline Chest radiograph & \\
\hline Chest CT & Within 1 year \\
\hline RUQ US & $\begin{array}{l}\text { Cystic fibrosis and alpha-one antitrypsin patients; } \\
\text { rule out portal hypertension }\end{array}$ \\
\hline Sinus CT & Cystic Fibrosis patients \\
\hline Full PFT's & Within 6 months \\
\hline Updated Spirometry & Within 6 months \\
\hline 6 Minute Walk Test & Within 6 months \\
\hline Lung Allocation Score Components & $\begin{array}{l}\text { If applicable (h/o autoimmune disease, symptoms of } \\
\text { delay) }\end{array}$ \\
\hline $\begin{array}{l}\text { Esophageal manometry \& gastric } \\
\text { emptying }\end{array}$ & Within 1 year \\
\hline PPD &
\end{tabular}

Figure 14: Lung pre-transplant evaluation checklist. The figure enlists few key tests performed to ensure appropriate patient selection and minimize post-transplant complications such as infection and organ failure. Some tests are performed based on the underlying diseases such as cystic fibrosis, autoimmune disease, etc. [ECHO = Echocardiography, EKG = Electrocardiogram, RUQ US = Right upper quadrant US, PPD = Purified protein derivative test for TB]

cystic fibrosis and autoimmune disease [Figure 14].

\section{Conclusions}

Post-lung transplant complications may have a wide spectrum of complex overlapping imaging appearances and these may frequently co-exist. Correlation of the clinicoradiological features with time course since transplantation significantly helps in narrowing the differential diagnosis. Low threshold for performing CT is advised, as it can detect and characterize infection early in this immunosuppressed population as well as aids in directing attempts at tissue biopsy. Tailoring of CT protocols in patients with suspected chronic allograft dysfunction or suspected anastomotic complications increases the yield of imaging in defining accurate diagnosis. Imaging can be critical to patient management if there is a concern for more rare complications of transplant, such as torsion, dehiscence, size mismatch, and airway complications.

\section{References}

1. De Perrot M, Chaparro C, McRae K, Waddell TK, Hadjiliadis D, Singer LG, et al. Twenty-year experience of lung transplantation at a single center: Influence of recipient diagnosis on long-term survival. J Thorac Cardiovasc Surg 2004;127:1493-501.

2. Hadjiliadis D, Chaparro C, Gutierrez C, Steele MP, Singer LG, Davis RD, et al. Impact of lung transplant operation on bronchiolitis obliterans syndrome in patients with chronic obstructive pulmonary disease. Am J Transplant 2006;6:183-9.

3. Weiss ES, Allen JG, Merlo CA, Conte JV, Shah AS. Factors indicative of long-term survival after lung transplantation: A review of 836 10-year survivors. J Heart Lung Transplant 2010;29:240-6.

4. Christie JD, Edwards LB, Kucheryavaya AY, Aurora P, Dobbels F, Kirk R, et al. The Registry of the International Society for Heart

and Lung Transplantation: Twenty-seventh official adult lung and heart-lung transplant report--2010. J Heart Lung Transplant 2010;29:1104-18.

5. Krishnam MS, Suh RD, Tomasian A, Goldin JG, Lai C, Brown K, et al. Postoperative complications of lung transplantation: Radiologic findings along a time continuum. Radiographics 2007;27:957-74.

6. Mason DP, Batizy LH, Wu J, Nowicki ER, Murthy SC, McNeill AM, et al. Matching donor to recipient in lung transplantation: How much does size matter? J Thorac Cardiovasc Surg 2009;137:1234-40. e1.

7. Kuno R, Kanter KR, Torres WE, Lawrence EC. Single lung transplantation followed by contralateral bullectomy for bullous emphysema. J Heart Lung Transplant 1996;15:389-94.

8. Bittner HB, Dunitz J, Hertz M, Bolman MR $3^{\text {rd }}$, Park SJ. Hyperacute rejection in single lung transplantation--case report of successful management by means of plasmapheresis and antithymocyte globulin treatment. Transplantation 2001;71:649-51.

9. De Jesus Peixoto Camargo J, Marcantonio Camargo S, Marcelo Schio S, Noguchi Machuca T, Adélia Perin F. Hyperacute rejection after single lung transplantation: A case report. Transplant Proc 2008;40:867-9.

10. Frost AE, Jammal CT, Cagle PT. Hyperacute rejection following lung transplantation. Chest 1996;110:559-62.

11. Montefusco CM, Veith FJ. Lung transplantation. Surg Clin North Am 1986;66:503-15.

12. Kundu S, Herman SJ, Winton TL. Reperfusion edema after lung transplantation: Radiographic manifestations. Radiology 1998;206:75-80.

13. Ferrer J, Roldan J, Roman A, Bravo C, Monforte V, Pallissa E, et al. Acute and chronic pleural complications in lung transplantation. J Heart Lung Transplant 2003;22:1217-25.

14. Herridge MS, de Hoyos AL, Chaparro C, Winton TL, Kesten S, Maurer JR. Pleural complications in lung transplant recipients. J Thorac Cardiovasc Surg 1995;110:22-6.

15. Ahmad S, Shlobin OA, Nathan SD. Pulmonary complications of lung transplantation. Chest 2011;139:402-11.

16. King-Biggs MB. Acute pulmonary allograft rejection. Mechanisms, diagnosis, and management. Clin Chest Med 1997;18:301-10.

17. Kundu S, Herman SJ, Larhs A, Rappaport DC, Weisbrod GL, Maurer J, et al. Correlation of chest radiographic findings with biopsy-proven acute lung rejection. J Thorac Imaging 1999;14:178-84.

18. Fuehner T, Simon A, Dierich M, Dewall C, Laenger F, Pletz MW, et al. Indicators for steroid response in biopsy proven acute graft rejection after lung transplantation. Respir Med 2009;103:1114-21.

19. Alvarez A, Algar J, Santos F, Lama R, Aranda JL, Baamonde C, et al. Airway complications after lung transplantation: A review of 151 anastomoses. Eur J Cardiothorac Surg 2001;19:381-7.

20. McAdams HP, Palmer SM, Erasmus JJ, Patz EF, Connolly JE, Goodman PC, et al. Bronchial anastomotic complications in lung transplant recipients: Virtual bronchoscopy for noninvasive assessment. Radiology 1998;209:689-95.

21. Ng YL, Paul N, Patsios D, Walsham A, Chung TB, Keshavjee S, et al. Imaging of lung transplantation: Review. AJR Am J Roentgenol 2009;192 Suppl 3:S1-19.

22. Boiselle PM, Feller-Kopman D, Ashiku S, Weeks D, Ernst A. Tracheobronchomalacia: Evolving role of dynamic multislice helical CT. Radiol Clin North Am 2003;41:627-36.

23. Lumsden AB, Anaya-Ayala JE, Birnbaum I, Davies MG, Bismuth J, Cheema ZF, et al. Robot-assisted stenting of a high-grade anastomotic pulmonary artery stenosis following single lung transplantation. J Endovasc Ther 2010;17:612-6. 
24. Collins J, Müller NL, Kazerooni EA, Paciocco G. CT findings of pneumonia after lung transplantation. AJR Am J Roentgenol 2000;175:811-8.

25. Paraskeva M, Bailey M, Levvey BJ, Griffiths AP, Kotsimbos TC, Williams TP, et al. Cytomegalovirus replication within the lung allograft is associated with bronchiolitis obliterans syndrome. Am J Transplant 2011;11:2190-6.

26. Morrish WF, Herman SJ, Weisbrod GL, Chamberlain DW. Bronchiolitis obliterans after lung transplantation: Findings at chest radiography and high-resolution CT. The Toronto Lung Transplant Group. Radiology 1991;179:487-90.

27. Estenne M, Maurer JR, Boehler A, Egan JJ, Frost A, Hertz M, et al. Bronchiolitis obliterans syndrome 2001: An update of the diagnostic criteria. J Heart Lung Transplant 2002;21:297-310.

28. Verleden GM, Raghu G, Meyer KC, Glanville AR, Corris P. A new classification system for chronic lung allograft dysfunction. J Heart Lung Transplant 2014;33:127-33.

29. Lee ES, Gotway MB, Reddy GP, Golden JA, Keith FM, Webb WR. Early bronchiolitis obliterans following lung transplantation: Accuracy of expiratory thin-section CT for diagnosis. Radiology 2000;216:472-7.

30. Konen E, Gutierrez C, Chaparro C, Murray CP, Chung T, Crossin J, et al. Bronchiolitis obliterans syndrome in lung transplant recipients: Can thin-section CT findings predict disease before its clinical appearance? Radiology 2004;231:467-73.

31. Arakawa H, Kurihara Y, Niimi H, Nakajima Y, Johkoh T, Nakamura $\mathrm{H}$ Bronchiolitis obliterans with organizing pneumonia versus chronic eosinophilic pneumonia: High-resolution CT findings in 81 patients. AJR Am J Roentgenol 2001;176:1053-8.

32. Paranjothi S, Yusen RD, Kraus MD, Lynch JP, Patterson GA, Trulock EP. Lymphoproliferative disease after lung transplantation: Comparison of presentation and outcome of early and late cases. J Heart Lung Transplant 2001;20:1054-63.

33. Collins J, Hartman MJ, Warner TF, Müller NL, Kazerooni EA, McAdams HP, et al. Frequency and CT findings of recurrent disease after lung transplantation. Radiology 2001;219:503-9.

34. Kazerooni EA, Cascade PN, Gross BH. Transplanted lungs: Nodules following transbronchial biopsy. Radiology 1995;194:209-12.

35. Baroni RH, Ashiku S, Boiselle PM. Dynamic CT evaluation of the central airways in patients undergoing tracheoplasty for tracheobronchomalacia. AJR Am J Roentgenol 2005;184:1444-9.

Cite this article as: Madan R, Chansakul T, Goldberg HJ. Imaging in lung transplants: Checklist for the radiologist. Indian J Radiol Imaging 2014;24:318-26.

Source of Support: Nil, Conflict of Interest: None declared. 